\title{
Adaptation of a 3-D Quadrupole Ion Trap for Dipolar DC Collisional Activation
}

\author{
Boone M. Prentice, ${ }^{1}$ Robert E. Santini, ${ }^{2}$ Scott A. McLuckey ${ }^{1}$ \\ ${ }^{1}$ Department of Chemistry, Purdue University, West Lafayette, IN 47907-2084, USA \\ ${ }^{2}$ The Jonathan Amy Facility for Chemical Instrumentation, Purdue University, West Lafayette, IN, USA
}

\begin{abstract}
Means to allow for the application of a dipolar DC pulse to the end-cap electrodes of a threedimensional (3-D) quadrupole ion trap for as short as a millisecond to as long as hundreds of milliseconds are described. The implementation of dipolar DC does not compromise the ability to apply AC waveforms to the end-cap electrodes at other times in the experiment. Dipolar DC provides a nonresonant means for ion acceleration by displacing ions from the center of the ion trap where they experience stronger if electric fields, which increases the extent of micro-motion. The evolution of the product ion spectrum to higher generation products with time, as shown using protonated leucine enkephalin as a model protonated peptide, illustrates the broad-band nature of the activation. Dipolar DC activation is also shown to be effective as an ion heating approach in mimicking high amplitude short time excitation (HASTE)/pulsed Q dissociation $(P Q D)$ resonance excitation experiments that are intended to enhance the likelihood for observing low $\mathrm{m} / \mathrm{z}$ products in ion trap tandem mass spectrometry.
\end{abstract}

Key words: Quadrupole ion trap, Dipolar DC, Ion trap collisional activation

\section{Introduction}

$\mathrm{T}$ he activation of gaseous ions to induce fragmentation is a key process in tandem mass spectrometry. Collisioninduced dissociation (CID) resulting from energetic collisions between accelerated ions and a thermal neutral target is the most widely used means for the primary structural characterization of ions [1, 2]. All common forms of tandem mass spectrometers are capable of generating product ion spectra from CID, although collision conditions (i.e., collision energies, numbers of collisions, nature of the bath gas, etc.) can vary widely from one instrument geometry to another [3, 4]. In the case of the quadrupole ion trap, CID is generally effected by accelerating precursor ions through the application of a supplemental AC signal to the end-cap

Electronic supplementary material The online version of this article (doi:10.1007/s13361-011-0183-z) contains supplementary material, which is available to authorized users.

Correspondence to: Scott A. McLuckey; e-mail: mcluckey@purdue.edu electrodes in resonance with their fundamental secular frequency $[5,6]$. This is often referred to as 'resonance excitation'. The AC voltage serves to increase the amplitude of the ion's harmonic oscillation in the trap, thereby increasing its maximum displacement from the trap's center. As displacement from the center of the ion trap increases, the ions experience higher rf field strengths associated with the main trapping voltage of the electrodynamic trap. The ions can undergo increased 'rf-heating', as reflected by the amplitude of the rf ripple superimposed upon the secular motion of the ion. Goeringer et al. have recently commented that this effect can be the major contributor to increasing the ion internal energy when collisions are probable [7], as is the case with ion traps operated in the presence of a relatively high pressure (e.g., 1-10 mTorr) bath gas. The supplemental AC signal is usually applied for $1-100 \mathrm{~ms}$ such that hundreds of collisions can occur over the many cycles of the secular motion (precursor ion secular frequencies of tens to hundreds of $\mathrm{kHz}$ are usual in most ion trap CID experiments). Under these conditions, polyatomic ion internal energies reach a steady state condition where ion heating 
and relaxation rates are comparable, which is a characteristic of a so-called 'slow heating' method [8,9].

Forms of ion acceleration in ion traps other than resonance excitation have been described for CID. For example, long DC pulses (i.e., tens of milliseconds) applied to the ring-electrode of the ion trap (i.e., quadrupolar DC) have been used to move ions close to a stability boundary where rf-heating can take place in a technique referred to as 'boundary-activated dissociation' [10]. However, the use of DC pulses applied to the ring electrode introduces a nonzero a-value and changes the secular frequency of the ion [11]. Wang and co-workers have reported simulations and experimental data using low frequency $\mathrm{AC}$ waveforms applied to the end-cap electrodes to effect nonresonant ion acceleration [12]. Ion activation using the low frequency AC waveform was attributed to energetic collisions associated with the oscillatory motion of precursor ions in response to the change in dipolar direction of the applied AC. Fast DC pulses (e.g., on the order of microseconds), applied either to the ring electrode (i.e., quadrupolar DC) or to the end-caps (i.e., in dipolar fashion), have been described for the purposes of CID [13], surface-induced dissociation [14], phase-locking [15], and DC tomography experiments [16]. Plass has characterized the effects of the application of dipolar DC on ion motion via simulations [17].

Tolmachev et al. have reported the use of relatively long dipolar DC pulses (i.e., 1-100 ms) in a linear ion trap coupled with a Fourier-transform ion cyclotron resonance mass spectrometer for activation purposes [18], whereby a dipolar potential is applied across a pair of opposing rods of a quadrupole array during an ion storage period. Ion activation with this approach arises from the increased ion velocities due to the enhanced ripple motion arising from the displacement of the ions to regions of the ion trap with higher rf quadrupolar fields. Tolmachev et al. presented a model to relate operating conditions to the 'effective internal temperatures' to which the precursor ion can be elevated [18]. We recently described the application of dipolar DC in a quadrupole collision cell of a quadrupole/time-of-flight tandem mass spectrometer [19] in both ion trapping and transmission modes. In the latter mode, a dipolar DC potential is applied as ions are continuously transmitted through the collision cell to the time-of-flight mass analyzer (i.e., there are no ion trapping steps). We also recently reported the application of a monopolar DC potential to an end-cap electrode of a three-dimensional (3-D) ion trap [20] and noted the observation of CID under some conditions. Under monopolar DC conditions, however, fragment ions are formed with non-zero a-values, which can lead to discrimination against high mass product ions generated from multiply charged precursor ions.

Dipolar DC as a means for ion acceleration has characteristics that can make it complementary to conventional single-frequency resonance excitation. First, unlike ACinduced ion activation, the DC activation method lacks a resonance condition and is thus a broadband technique, somewhat similar in principal but different in implementation to stored waveform inverse Fourier transform (SWIFT) pulses [21]. According to Tolmachev's model [18], under a fixed set of conditions all polyatomic ions reach the same internal temperature regardless of mass and charge state. In this respect, the dipolar DC approach is analogous to heating the bath gas and ion trap such that the precursor ion and all product ions are maintained at the same temperature (i.e., a thermal dissociation experiment $[22,23])$. Like the continuous wave infrared multi-photon dissociation (IRMPD) experiment in the ion trap [24], the ion trap DDC experiment exposes both the precursor ion and product ions to continuous activation. This stands in contrast with singlefrequency resonance excitation in which first generation product ions are actively cooled by collisions with the bath gas. In this work, we describe the implementation of dipolar DC to the end-caps of a 3-D ion trap that does not compromise the use of AC wave-forms for resonance excitation or resonance ejection. We illustrate its use with leucine enkephalin and demonstrate a DDC analog to 'fast excitation CID' [25], 'pulsed Q dissociation' (PQD) [26], or 'high amplitude short time excitation' HASTE [27]. These terms refer to a method that employs a short high amplitude dipolar AC pulse at a relatively high q-value to accelerate and heat a precursor ion of interest that is, in PQD and HASTE techniques, immediately followed by an ion trapping period at a lower q-value that allows for the capture of low $\mathrm{m} / \mathrm{z}$ products from precursor ions that fragment after the excitation period. Other fast resonant activation methods including dynamic CID [28] and pulsed q dynamic CID [29] have been used in similar fashions to activate ions whilst simultaneously varying ion q-value.

\section{Experimental}

\section{Instrumentation}

All experiments were performed on a dual source Finnigan Ion Trap Mass Spectrometer (ITMS; ThermoFinnigan Corp., San Jose, CA, USA), modified to allow for ion/ion reactions, as previously described (Figure 1) [30]. Briefly, ions from one of two sources can be sequentially injected axially into the ion trap by means of a DC turning quadrupole. Only one ion source is depicted in Figure 1 as no ion/ion reactions were involved in this work. The injection and timing are controlled using Ion Catcher Mass Spectrometer software (ICMS Ion Trap Software ver. 2.20; University of Florida).

Application of the DDC pulse was controlled via a transistor-transistor logic (TTL) trigger under software control. The TTL trigger controlled a custom-built switchbox previously described [30] as well as the initiation of a DC pulse from an Agilent 8114A high voltage programmable pulse generator (Agilent Technologies, Santa Clara, CA, UA). The switchbox provided multiple high isolation single pole double throw (SPDT) functions for various inputs as indicated in Figure 1. The pulse generator is 


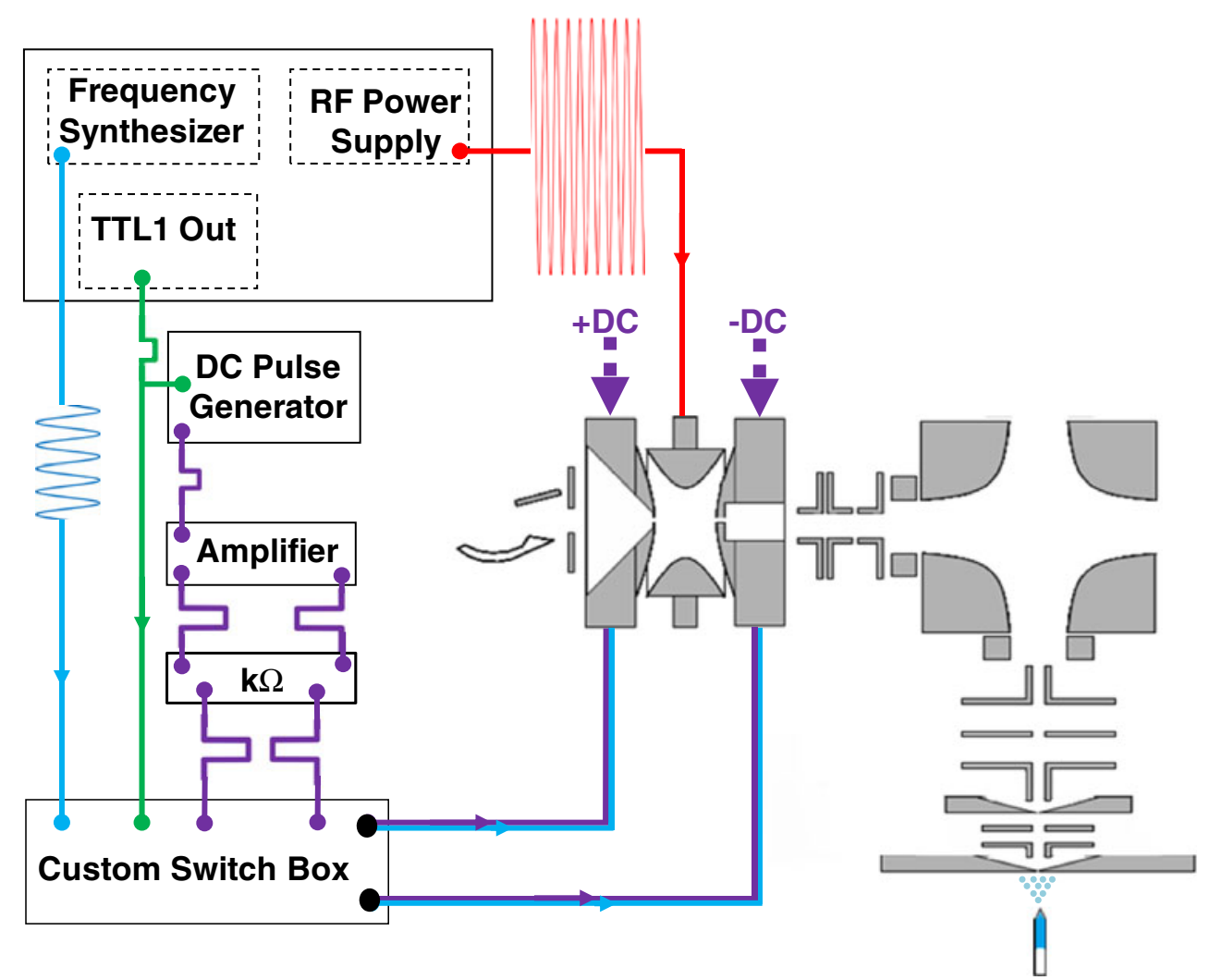

Figure 1. Schematic (not to scale) of modified dual source 3-D ion trap mass spectrometer [30]. '+DC' and '-DC' define the dipolar DC potentials applied to the end-cap electrodes

programmed such that the DC pulse is internally initiated by the TTL trigger, but output of the pulse from the pulse generator is delayed $1.5 \mathrm{~ms}$. This is done to minimize ringing artifacts associated with the DC pulse step response. The DC pulse from the pulse generator is then run through custom built boosters based on APEX PA85 high voltage modules (Cirrus Logic, Inc., Austin, TX, USA), which provided two amplified outputs opposite in polarity and equal in magnitude. This gave a range of roughly 0 to $350 \mathrm{~V}$ DDC with an absolute resolution of roughly \pm 1 volt. The APEX circuits were powered by two Kikisui PMC350-0.2A power supplies (Kikisui America, Inc., Burlingame, CA, USA), which acted as high voltage rail power supplies (operated at $\pm 225 \mathrm{~V}$ ) and a three-output Agilent E3630A power supply (Agilent Technologies, Santa Clara, CA, USA), which acted as a low voltage rail power supply (operated at $\pm 15 \mathrm{~V}$ ). The outputs from the high voltage amplifier were applied to the switchbox, which was toggled by the TTL trigger as mentioned between the DC pulse channels and the channels for supplemental dipolar AC as provided from the original Finnigan electronics. Simple resistive isolation networks were developed in series between each output of the APEX amplifier and the switchbox (two resistors whose values of 820 and $890 \mathrm{k} \Omega$, totaling $1710 \mathrm{k} \Omega$, were empirically determined). These were inserted in order to prevent the inherent capacitive coupling of the drive $\mathrm{rf}$ to the end-cap electrodes from shunting back to the amplifying circuitry, while still maintaining adequate DDC pulse rise and fall times. In the absence of this protection, the DDC pulse as well as the rf field within the ion trap would become distorted.

This configuration is capable of delivering stable pulses ranging from one to hundreds of milliseconds. The rise time of the DC pulse is tens of microseconds, which is comparable to the period of ion oscillation inside the trap $(10-100 \mu \mathrm{s})$, but much faster than the rise time of the drive $\mathrm{rf}(\sim 100 \mu \mathrm{s})$. The fall time of the DC pulse is roughly 200 to $300 \mu \mathrm{s}$, which is slightly slower than that of the drive rf $(\sim 100 \mu \mathrm{s})$. This timing is important for two reasons. The rise and fall time of the pulse should be slower than the ion motion so that the phase angle of the ions when the DC pulse is initiated is irrelevant. If the rise time of the pulse were much shorter than the period of ion oscillation (i.e., it could be viewed as instantaneous), the ion population may be initially displaced by a magnitude substantially larger or smaller than the final equilibrium displacement, as determined by the phase angle of ion oscillation. By having a relatively slow rising pulse, there is no initial impulsive effect on the ion population that is dependent on oscillation phase. Conversely, as the DC pulse fall time is slightly too slow, (i.e., longer than the fall time of the drive rf), a residual dipolar DC voltage can extend into a subsequent step of the scan function. The latter situation can be problematic when the drive $\mathrm{rf}$ is reduced immediately after the DC pulse 
because the residual dipolar DC could eject the ions. This is accounted for by letting the pulse decay for roughly 0.5 to $1 \mathrm{~ms}$ before reducing the drive rf (Figure 2).

A second pulse configuration has been realized which has pulse rise times of roughly $1 \mu \mathrm{s}$. This set up is also capable of outputting $0-350 \mathrm{~V}$ DDC, $\pm 1 \mathrm{~V}$. This arrangement uses custom-built band reject PI-based notch filters in order to eliminate the shunting of the drive $\mathrm{rf}$ back to the amplifying circuitry. Placing these filters in series between each output of the APEX amplifier and the trap eliminates the need for the time sharing functions provided by the switchbox. In this method, the supplemental AC signal is modulated through the APEX circuitry unamplified and applied to the end-caps in a dipolar fashion. This provides the ability to apply simultaneous AC and DDC waveforms.

\section{Results}

Like the resonance excitation approach to ion acceleration in the ion trap, DDC excitation is a slow heating method. After a transition period from the initial ion internal energy distribution, which is close to the bath gas temperature, the precursor ions reach an elevated steady state internal energy distribution [8]. Once the ions have reached the steady state internal energy distribution, pseudo-first order kinetics are observed for a common set of precursor ions (i.e., a population with the same structure and kinetic stability).

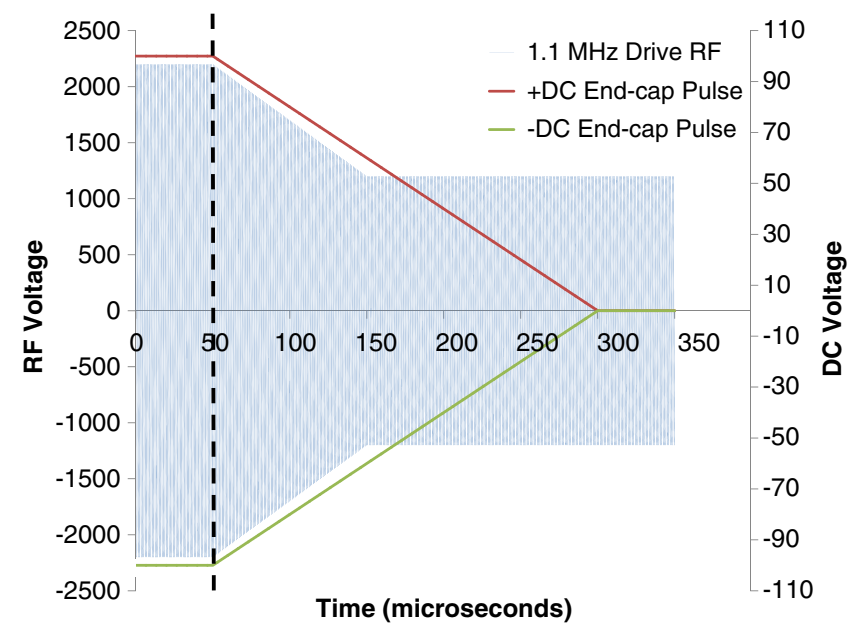

Figure 2. Diagram illustrating fall times of the drive if and DDC pulse. The dotted line indicates the end of a segment in the scan function with a specific LMCO and DDC applied and the beginning of a subsequent segment with a lower LMCO and no DDC applied. Note that the DC pulse fall time is slightly longer than that of the drive rf. By this, a residual DDC voltage can extend into a subsequent step of the scan function. This can be problematic when the drive if is reduced immediately after the $\mathrm{DC}$ pulse because the residual dipolar DC could eject the ions. This is accounted for by letting the pulse decay for roughly 0.5 to $1 \mathrm{~ms}$ before reducing the drive if
For a fixed pressure, bath gas, ion trap geometry, and drive frequency, the major variables in a DDC activation experiment are DDC amplitude, $\mathrm{V}_{\mathrm{DDC}}$, the amplitude of the $\mathrm{rf}$ voltage, $\mathrm{V}_{\mathrm{rf}}$, and the duration of the activation period. The $\mathrm{V}_{\mathrm{DDC}} / \mathrm{V}_{\mathrm{rf}}$ ratio determines the final steadystate internal energy distribution [18], while the activation time determines the period over which the ion population can evolve at the elevated 'temperature' associated with the ion acceleration. A key difference between DDC activation and resonance excitation is that the product ions are nominally maintained at the same 'temperature' as the precursor ions in the former whereas the product ions are cooled to the temperature of the bath gas in the latter [31]. Hence, the identities and relative abundances in product ion spectra obtained via resonance excitation tend to be invariant with activation time when the bath gas is at room temperature [32], whereas first-generation product ions are more likely to continue to fragment under DDC activation. The $[\mathrm{M}+\mathrm{H}]^{+}$ion of leucine enkephalin, which is a widely used thermometer ion in mass spectrometry [33], as well as the $[\mathrm{M}+\mathrm{Na}]^{+}$ion were isolated and simultaneously exposed to DDC for varying degrees of time to illustrate the time evolution of the product ion spectra. Figure 3 compares results obtained prior to application of DDC (Figure 3(a)) with those obtained after application of $36 \mathrm{~V}$ DDC at $\mathrm{V}_{\mathrm{rf}}=1250 \mathrm{~V}$ (low mass cut-off $=100 \mathrm{Da}, \mathrm{q}_{\mathrm{z}}=0.16$ ) for $2 \mathrm{~ms}$ (Figure 3 (b)), $10 \mathrm{~ms}$ (Figure 3(c)), and $20 \mathrm{~ms}$ (Figure 3(d)).

Three general observations regarding DDC activation can be drawn from the comparison of Figure 3. First, it is clear that the abundances of the product ions increase with activation time relative to those of the precursor ions. The precursor ion dissociation rate can be determined by monitoring its disappearance with time. Figure S-1 in Supplemental Information summarizes a series of kinetic plots obtained at various values of $\mathrm{V}_{\mathrm{DDC}}$ at constant $\mathrm{V}_{\mathrm{rf}}$ and vice versa. Second, it is also clear that the $[\mathrm{M}+\mathrm{Na}]^{+}$ion fragments at a much lower rate under the conditions of Figure 3 than does the $[\mathrm{M}+\mathrm{H}]^{+}$ion, which is consistent with the known relative stabilities of these forms of ionized leucine enkephalin [31]. Third, the abundances of various product ions change relative to one another with time. The changes are consistent with contributions from consecutive fragmentation. The clearest indication of sequential fragmentation can be seen in the decrease in the $b_{4} / a_{4}$ product ion ratio with increasing time. It is well-known that the $a_{4}$ ion is the major decomposition product of the $b_{4}$ ion $[31,34]$. This behavior stands in contrast to single-frequency resonance excitation of protonated leucine enkephalin in room temperature helium, from which the $\mathrm{b}_{4} / \mathrm{a}_{4}$ product ion ratio is essentially invariant. The third observation is a reflection of the broad-band nature of DDC activation. Due to the much greater kinetic stability of the $[\mathrm{M}+\mathrm{Na}]^{+}$ ion (i.e., a higher activation barrier to dissociation than the $[\mathrm{M}+\mathrm{H}]^{+}$ion), very little fragmentation of this 


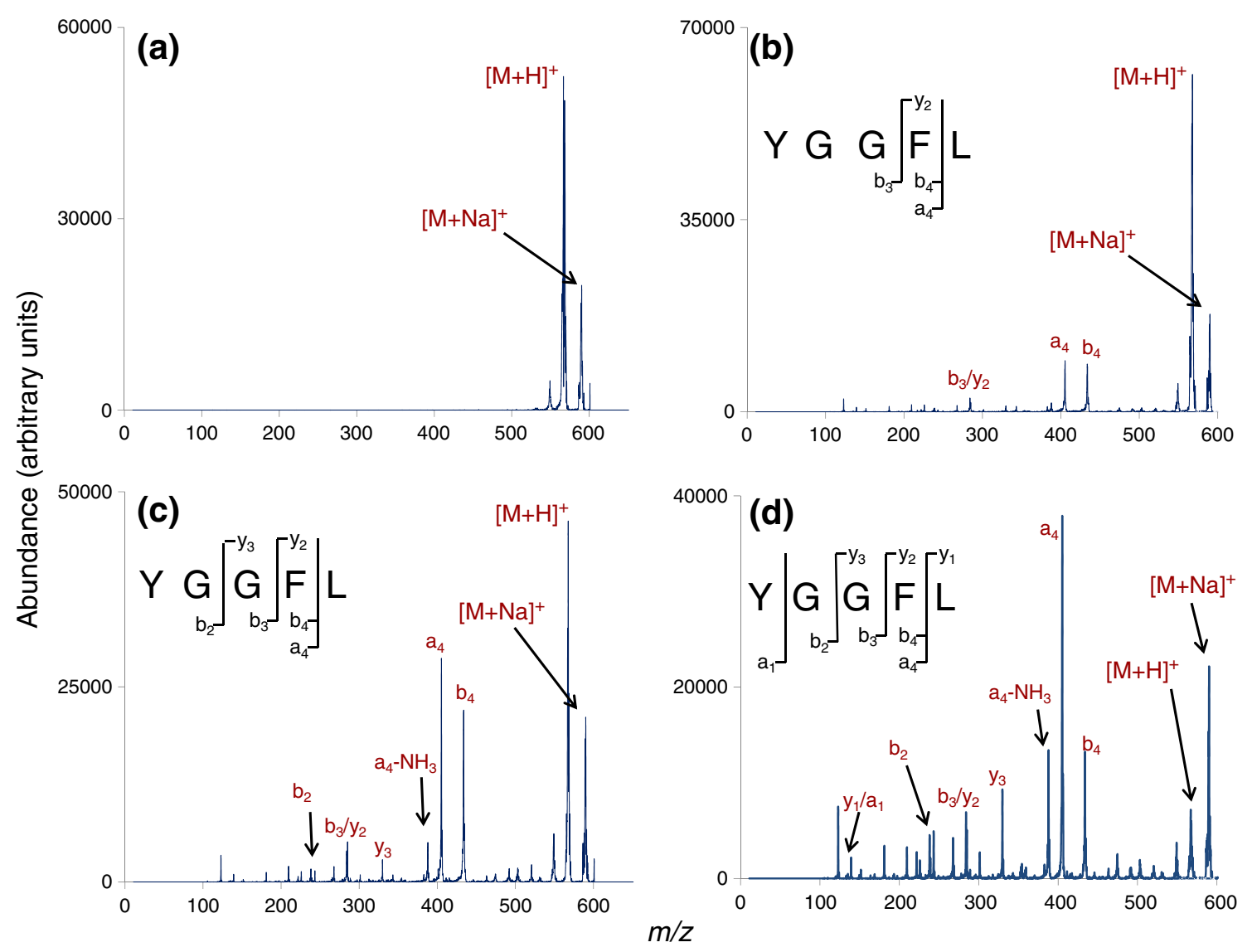

Figure 3. Protonated leucine enkephalin (a) without activation and activation performed with $36 \mathrm{~V}$ DDC at a LMCO of 100 Da $\left(q_{z}=0.16\right)$ for (b) $2 \mathrm{~ms}$, (c) $10 \mathrm{~ms}$, and (d) $20 \mathrm{~ms}$

precursor ion was noted in the spectrum under these conditions. An illustration of simultaneous dissociation of two precursor ion populations (i.e., the $[\mathrm{M}+\mathrm{H}]^{+}$ions of YGGFL and YGGFLK) is shown in Figure S-2 in Supplemental Information.

The characteristics noted for DDC activation suggest that it should be possible to use it as a non-resonant means for heating ions relatively rapidly under conditions of relatively high $\mathrm{V}_{\mathrm{rf}}$, which minimizes parent ion loss by maintaining a high q-value for the precursor ion during ion acceleration, and then to drop the $\mathrm{V}_{\mathrm{rf}}$ upon cessation of ion acceleration to allow for capture of relatively low $\mathrm{m} / \mathrm{z}$ product ions that are generated after the excitation period (typically $<2 \mathrm{~ms}$ ). When resonance excitation is used for ion acceleration, this procedure has been termed PQD [26] or HASTE [27]. Figure 4(a) shows the results of a PQD/HASTE experi- (a)

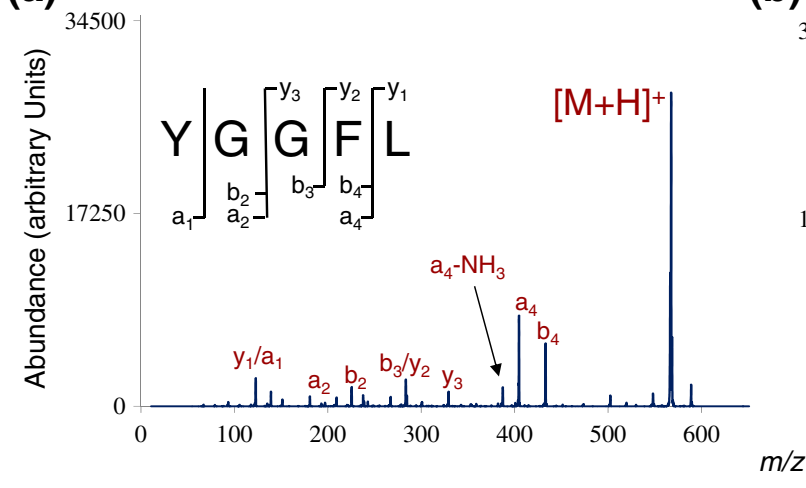

(b)

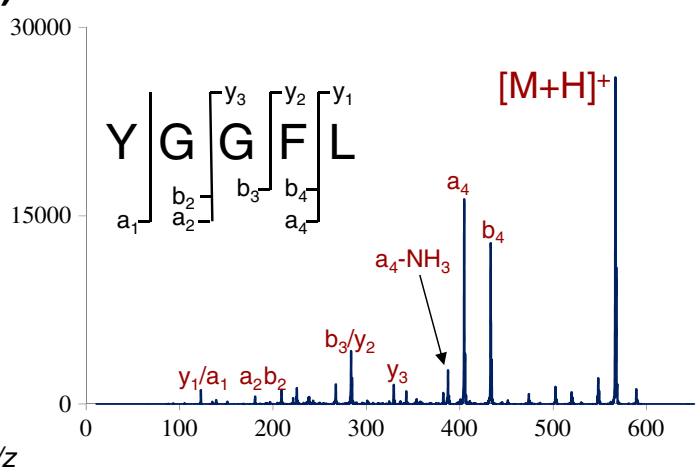

Figure 4. (a) Resonance excitation of protonated YGGFL for $1 \mathrm{~ms}$ at an excitation amplitude of $1.9 \mathrm{~V}_{p-p}$ at $\mathrm{q}_{\mathrm{z}}=0.28$ followed by a $10 \mathrm{~ms}$ storage period at $\mathrm{q}_{\mathrm{z}}=0.08$ prior to mass analysis, and (b) DDC activation of protonated YGGFL for 1 ms with an excitation amplitude of $116 \mathrm{~V}$ at $\mathrm{q}_{\mathrm{z}}=0.28$ followed by a $10 \mathrm{~ms}$ storage period at $\mathrm{q}_{\mathrm{z}}=0.08$ prior to mass analysis 
ment for protonated YGGFL using a resonance excitation amplitude of $1.9 \mathrm{~V}_{p-p}$ for $1 \mathrm{~ms}$ at a precursor ion q-value $=0.28$ (low mass cut-off $=170 \mathrm{Da}$ ) followed by a $10 \mathrm{~ms}$ storage period at $\mathrm{q}_{\mathrm{z}}=0.08$ (low mass cut-off $=50 \mathrm{Da}$ ) prior to mass analysis. Figure 4(b) shows the DDC analogy in which a dipolar DC voltage of $116 \mathrm{~V}$ was applied for $1 \mathrm{~ms}$ at a q-value of 0.28 followed by a $10 \mathrm{~ms}$ storage period at $\mathrm{q}_{\mathrm{z}}=0.08$ prior to mass analysis. The two experiments give quite similar results with both showing extensive fragmentation and the presence of low $\mathrm{m} / \mathrm{z}$ fragments, specifically the $a_{2}, a_{1}, y_{1}$, and $b_{1}$ fragments.

\section{Conclusions}

Application of a dipolar DC voltage to the end-cap electrodes of a quadruple ion trap leads to a broadband, continuous heating of stored ions. We describe here a means for implementing DDC without compromising the ability to apply tailored wave-forms to the end-cap electrodes instead of, or in conjunction with, the application of DDC. An in-depth evaluation of the strengths and limitations of DDC in the 3-D ion trap relative to ACwaveform approaches is under way. However, it is already clear that DDC has characteristics that can complement those of single-frequency resonance excitation. In addition to demonstrating sequential fragmentation that can be driven by the application of DDC, we also show that a relatively high DDC voltage applied for $1 \mathrm{~ms}$ can be used as the excitation step of a PQD/HASTE experiment. Hence, DDC provides a nonresonant (i.e., non-mass-selective) means for ion acceleration using the PQD/HASTE strategy for generating and observing relatively low $\mathrm{m} / \mathrm{z}$ products in an ion trap.

\section{Acknowledgments}

The authors acknowledge Jim Zimmerman and Rob Oglesbee of the Jonathan Amy Facility for Chemical Instrumentation for their support. This work was sponsored by the National Institutes of Health under grant no. GM45372.

\section{References}

1. Wells, J.M., McLuckey, S.A.: Collision-induced dissociation (CID) of peptides and proteins. Methods Enzymol. 402, 148-185 (2005)

2. McLuckey, S.A., Mentinova, M.: Ion/neutral, ion/electron, ion/photon, and ion/ion interactions in tandem mass spectrometry: Do we need them all? Are they enough? J. Am. Soc. Mass Spectrom. 22, 3-12 (2011)

3. McLuckey, S.A.: Principles of collisional activation in analytical mass spectrometry. J. Am. Soc. Mass Spectrom. 3, 599-614 (1992)

4. McLuckey, S.A., Goeringer, D.E.: Slow heating methods in tandem mass spectrometry. J. Mass Spectrom. 32, 461 (1997)

5. Louris, J.N., Cooks, R.G., Syka, J.E.P., Kelley, P.E., Stafford Jr., G.C., Todd, J.F.J.: Instrumentation, applications, and energy deposition in quadrupole ion-trap tandem mass spectrometry. Anal. Chem. 59, 16771685 (1987)
6. Wang, M., George III, J.E.: Technology progress and application in GC/ MS and GC/MS/MS. In: March, R.E., Todd, J.F.J. (eds.) Practical Aspects of Trapped Ion Mass Spectrometry, vol. V. CRC Press, Boca Raton, FL (2009). Chapter 15

7. Goeringer, D.E., Viehland, L.A., Danailov, D.M.: Prediction of collective characteristics for ion ensembles in quadrupole ion traps without trajectory simulations. J. Am. Soc. Mass Spectrom. 17, 889-902 (2006)

8. Goeringer, D.E., McLuckey, S.A.: Evolution of ion internal energy during collisional excitation in the Paul ion trap: A stochastic approach. J. Chem. Phys. 104, 2214-2221 (1996)

9. McLuckey, S.A., Goeringer, D.E.: Slow heating methods in tandem mass spectrometry. J. Mass Spectrom. 32, 461 (1997)

10. Paradisi, C., Todd, J.F.J., Vettori, U.: Boundary effects and collisional activation in a quadrupole ion trap. Org. Mass Spectrom. 27, 251-254 (1992)

11. Splendore, M., Lausevic, M., Lausevic, Z., March, R.E.: Resonant excitation and/or ejection of ions subjected to DC and RF fields in a commercial quadrupole ion trap. Rapid Commun. Mass Spectrom. 11, 228-233 (1997)

12. Wang, M., Schachterle, S., Wells, G.: Application of non-resonance excitation to ion trap tandem mass spectrometry and selected ejection chemical ionization. J. Am. Soc. Mass Spectrom. 7, 668676 (1996)

13. Lammert, S.A., Cooks, R.G.: Pulsed axial activation in the ion trap: A new method for performing tandem mass spectroscopy (MS/MS). Rapid Commun. Mass Spectrom. 6, 528-530 (1992)

14. Lammert, S.A., Cooks, R.G.: Surface-induced dissociation of molecular-ions in a quadrupole ion trap mass-spectrometer. J. Am. Soc. Mass Spectrom. 2, 487-491 (1991)

15. Jullian, R.K., Nappi, M., Weil, C., Cooks, R.G.: Multiparticlesimulation of ion motion in the ion trap mass spectrometer: resonant anddirect current pulse excitation. J. Am. Soc. Mass Spectrom. 6, 57-70 (1995)

16. Cooks, R.G., Cleven, C.D., Horn, L.A., Nappi, M., Weil, C., Soni, M. H., Julian, R.K.: Nondestructive detection of ions in a quadrupole ion trap using a DC pulse to force coherent ion motion: A simulation study. Int. J. Mass Spectrom. Ion Processes 146/147, 147-163 (1995)

17. Plass, W.R.: Theory of dipolar DC excitation and DC tomography in the rf quadrupole ion trap. Int. J. Mass Spectrom. 202, 175-197 (2000)

18. Tolmachev, A.V., Vilkov, A.N., Bogdanov, B., Păsa-Tolić, L., Masselon, C.D., Smith, R.D.: Collisional activation of ions in $\mathrm{rf}$ ion traps and ion guides: The effective ion temperature treatment. J. Am. Soc. Mass Spectrom. 15, 1616-1628 (2004)

19. Webb, I.K., Londry, F.A., McLuckey, S.A.: Implementation of dipolar DC CID in storage and transmission modes on a quadrupole/time-offlight tandem mass spectrometer. Rapid Commun. Mass Spectrom., submitted

20. Prentice, B.M., Xu, W., Ouyang, Z., McLuckey, S.A.: DC potentials applied to an end-cap electrode of a 3-D ion trap for enhanced $\mathrm{MS}^{n}$ functionality. Int. J. Mass Spectrom. Article in press, doi:10.1016

21. Julian, R.K., Cooks, R.G.: Broad-band excitation in the quadrupole iontrap mass spectrometer using shaped pulses created with the inverse Fourier transform. Anal. Chem. 65, 1827-1833 (1993)

22. Asano, K. G.; Goeringer, D. E.; McLuckey, S. A. Thermal dissociation in the quadrupole ion trap: Ions derived from leucine enkephalin. Int. $J$. Mass Spectrom. 185/186/187, 207-219 (1999).

23. Jue, A.L., Racine, A.H., Glish, G.L.: The effect of ion trap temperature on the dissociation of peptide ions in a quadrupole ion trap. Int. J. Mass Spectrom. 301, 74-83 (2011)

24. Brodbelt, J.S.: Shedding light on the frontier of photodissociation. $J$. Am. Soc. Mass Spectrom. 22, 197-206 (2011)

25. Murrell, J., Despeyroux, D., Lammert, S.A., Stephenson Jr., J.L., Goeringer, D.E.: "Fast excitation" CID in a quadrupole ion trap mass spectrometer. J. Am. Soc. Mass Spectrom. 14, 785-789 (2003)

26. Schwartz, J.C., Syka, J.E.P., Quarmby, S.T. Proceedings of the 53rd ASMS Conference on Mass Spectrometry and Allied Topics; San Antonio, TX, June 5-9 (2005)

27. Cunningham Jr., C., Glish, G.L., Burinsky, D.J.: High amplitude short time excitation: A method to form and detect low mass product ions in a quadrupole ion trap mass spectrometer. J. Am. Soc. Mass Spectrom. 17, 81-84 (2006)

28. Collin, O.L., Beier, M., Jackson, G.P.: Dynamic collision-induced dissociation of peptides in a quadrupole ion trap mass spectrometer. Anal. Chem. 79, 5468-5473 (2007) 
29. Laskay, U.A., Jackson, G.P.: Resonance excitation and dynamic collision-induced dissociation in quadrupole ion traps using higherorder excitation frequencies. Rapid Commun. Mass Spectrom. 22, 2342-2348 (2008)

30. Wells, J.M., Chrisman, P.A., McLuckey, S.A.: Dueling electrospray: Instrumentation to study ion/ion reactions of electrospray-generated cations and anions. J. Am. Soc. Mass Spectrom. 13, 614-622 (2002)

31. Goeringer, D.E., McLuckey, S.A.: Relaxation of internally excited high mass ions simulated under typical quadrupole ion trap storage conditions. Int. J. Mass Spectrom. 177, 163-174 (1998)

32. Asano, K.G., Goeringer, D.E., Butcher, D.J., McLuckey, S.A.: Bath gas temperature and the appearance of ion trap tandem mass spectra of high-mass ions. Int. J. Mass Spectrom. 190/191, 281-293 (1999)

33. Sztáray, J., Memboeuf, A., Drahos, L., Vékey, K.: Leucine enkephalina mass spectrometry standard. Mass Spectrom. Rev. 30, 298-320 (2011)

34. Jue, A.L., Racine, A.H., Glish, G.L.: The effect of ion trap temperature on the dissociation of peptide ions in a quadrupole ion trap. Int. J. Mass Spectrom. 301, 74-83 (2011) 